\title{
A NON-PERTURBATIVE TREATMENT OF THE GENERALIZED SU-SCHRIEFFER-HEEGER HAMILTONIAN ON A DIMER.
}

\author{
M. COCOCCIONI \\ Dipartimento di Fisica, Università di Pavia \\ Pavia, I-27100, Italy 1 \\ and \\ M. ACQUARONE \\ C.N.R.-G.N.S.M., Unita' I.N.F.M., Dipartimento di Fisica,Universita' di Parma \\ Parma, I-43100, Italy
}

\begin{abstract}
.
Starting from the Hamiltonian for a dimer which includes all the electronic and electron-phonon terms consistent with a non-degenerate orbital, by a sequence of displacement and squeezing transformation we obtain an effective polaronic Hamiltonian. The renormalized electronic interactions differ from the results of semiclassical or perturbative treatments. The properties of the variationally determined ground state of two particles in the orbital are discussed for variable dimer length in the adiabatic limit.
\end{abstract}

\section{Introduction.}

Thirty years ago, the electron-phonon interaction $\gamma_{i j} \equiv d t_{i j} / d\left(\mathbf{R}_{j}-\mathbf{R}_{i}\right)$, resulting from the modulation of the hopping amplitude $t_{i j}$ due to the displacement $u_{i}$ of the $i$-th site from its equilibrium position $\mathbf{R}_{i}$ in the lattice, was indicated as responsible of the BCS superconductivity in transition metals 1. Later on its relevance also to the physics of quasi-unidimensional conducting polymers [2] and layer compounds [3] was recognized. Interest in its effects, however, was rather sporadic until the seminal paper on dimerisation in unidimensional systems [4. Since then, the Hamiltonian

$$
H_{S S H}=\sum_{\langle i, j\rangle \sigma} \gamma_{i j}\left(c_{i \sigma}^{\dagger} c_{j \sigma}+c_{j \sigma}^{\dagger} c_{i \sigma}\right)\left(u_{j}-u_{i}\right)
$$

is known as the Su-Schrieffer-Heeger ( $\mathrm{SSH}$ ) model[5]. The suggestion was also advanced that it had to do with the bipolaronic superconductivity [6], a very exotic possibility at that time. Its effect in transition metals was reexamined in Ref. [7].

\footnotetext{
${ }^{1}$ Present address: International School for Advanced Studies, Via Beirut 2/4, Grignano (TS) I-34014, Italy
} 
All those issues became hot subjects of debate after the discovery of the hightemperature superconductors, promoting many investigations 8 on its possible effects in the cuprates. However, due to the impossibility of an exact solution on an infinite lattice, the study of $H_{S S H}$ has generally been limited to the quasi-classical approximation, treating $\left(u_{j}-u_{i}\right)$ as a $c$-number, and resulting in a modification of the hopping amplitude $t_{i j}$ linearly depending on $\gamma_{i j}$ : $t_{i j}^{*}=t_{i j}-\gamma_{i j}\left(u_{j}-u_{i}\right)$. A second- order perturbative treatment has been recently published [9] in the context of the equivalence between spinless SSH interaction term and the $X$ - $Y$ spinPeierls Hamiltonian, suggesting a quadratic dependence of $t_{i j}^{*}$ on $\gamma_{i j}$. On small size clusters the problem allows in principle for an explicit analytical solution with $\left(u_{j}-u_{i}\right)$ properly quantized. Here we shall present a non-perturbative study of a two-site model system (a dimer) where the assumed set of electron-phonon interactions generalizes $H_{S S H}$ by including other terms consistent with one electron in a non-degenerate orbital, as discussed in Ref.10.

\section{The model.}

Let us consider a general two-site electron-phonon Hamiltonian $H=H_{e l}+H_{p h}+$ $H_{e l-p h}$ where, in standard notation for a non-degenerate orbital 11]:

$$
\begin{aligned}
& H_{e l}=\epsilon \sum_{\sigma}\left(n_{1 \sigma}+n_{2 \sigma}\right)+\sum_{\sigma}\left[t+X\left(n_{1-\sigma}+n_{2-\sigma}\right)\right]\left(c_{1 \sigma}^{\dagger} c_{2 \sigma}+H . c .\right)+U\left(n_{1 \uparrow} n_{1 \downarrow}+n_{2 \uparrow} n_{2 \downarrow}\right) \\
& \quad+(V-J / 2) n_{1} n_{2}-2 J\left[S_{1}^{z} S_{2}^{z}+\frac{1}{2}\left(S_{1}^{+} S_{2}^{-}+H . c .\right)\right]+P\left(c_{1 \uparrow}^{\dagger} c_{1 \downarrow}^{\dagger} c_{2 \downarrow} c_{2 \uparrow}+H . c .\right) . \quad(2)
\end{aligned}
$$

and $H_{e l-p h}$ is the adiabatic Hamiltonian introduced in Ref.10, which generalizes the SSH Hamiltonian.

$$
H_{e p}=g_{\epsilon}^{(12)} \sum_{\sigma}\left(n_{1 \sigma}+n_{2 \sigma}\right)\left(u_{2}-u_{1}\right)+\gamma^{(12)} \sum_{\sigma}\left(c_{1 \sigma}^{\dagger} c_{2 \sigma}+c_{2 \sigma}^{\dagger} c_{1 \sigma}\right)\left(u_{2}-u_{1}\right) \text {. }
$$

For symmetry reasons, the deformation $u_{i}$ on site $i$ at $\mathbf{R}_{i}=\left[\left(-1^{i}\right) a / 2,0,0\right](i=1,2)$ has to be along the $x$-axis, therefore $u_{2}-u_{1}$ is the variation of the dimer length $a$, while $u_{1}+u_{2}=0$. By assuming a single phonon frequency $\Omega$, quantization is performed as usual by writing $u_{i}=L\left(b_{i}^{\dagger}+b_{i}\right)$ with $L=\sqrt{\hbar / 2 M \Omega}$. It is convenient to introduce the odd-parity phonon operators $d, d^{\dagger}$ according to $b_{1}^{(\dagger)}-b_{2}^{(\dagger)}=\sqrt{2} d^{(\dagger)}$. The even-parity operators do not couple to the electrons, and contribute to the energy a constant term. Then, after rescaling the coupling constants by $\hbar \Omega / \sqrt{2} L$, $H_{e p}$ reads:

$$
H_{e p}=\left[g_{\epsilon}^{(12)} \sum_{\sigma}\left(n_{1 \sigma}+n_{2 \sigma}\right)+\gamma^{(12)} \sum_{\sigma}\left(c_{1 \sigma}^{\dagger} c_{2 \sigma}+c_{2 \sigma}^{\dagger} c_{1 \sigma}\right)\right]\left(d^{\dagger}+d\right) .
$$

while free oscillator term reads $H_{p h}=\hbar \Omega\left(d^{\dagger} d+1 / 2\right)$ To obtain an effective electrononly Hamiltonian, describing fermions dressed by phonons, we perform on $H_{e l}+$ $H_{e p}+H_{p h}$ a unitary "displacement " transformation $\exp (\delta R)$, where (from now on we drop the site indexes on $g^{(12)}$ and $\gamma_{12}$ for short):

$$
R=\left[g\left(n_{1}+n_{2}\right)+\gamma\left(c_{1}^{\dagger} c_{2}+c_{2}^{\dagger} c_{1}\right)\right]\left(d^{\dagger}-d\right)=-R^{\dagger}
$$


with $\delta$ an up to now undetermined parameter. The "displaced" Bose operators $D_{i}^{(\dagger)} \equiv e^{\delta R} d^{(\dagger)} e^{-\delta R}$ are:

$$
D_{i}^{(\dagger)}=d_{i}^{(\dagger)}-\delta\left[g\left(n_{1}-n_{2}\right)+\gamma\left(c_{1}^{\dagger} c_{2}+c_{2}^{\dagger} c_{1}\right)\right]
$$

For the "displaced" Fermi operators $f_{i}^{\dagger} \equiv e^{\delta R_{\delta}} c_{i}^{\dagger} e^{-\delta R_{\delta}}$ we have that the equations of motion (EOM):

$$
\left.\begin{array}{l}
\partial f_{1}^{\dagger} / \partial \delta=\left(g f_{1 \sigma}^{\dagger}+\gamma f_{2 \sigma}^{\dagger}\right)\left(d^{\dagger}-d\right) \\
\partial f_{2}^{\dagger} / \partial \delta=\left(g f_{2 \sigma}^{\dagger}+\gamma f_{1 \sigma}^{\dagger}\right.
\end{array}\right)\left(d^{\dagger}-d\right)
$$

can be decoupled due to the finiteness of the system, and an analytical solution is obtained. Taking into account the boundary conditions for $\delta=0$, defining for short $B=\delta\left(d^{\dagger}-d\right)=-B^{\dagger}$ and indicating by $\mathrm{Ch}(x)$ and $\mathrm{Sh}(x)$ the hyperbolic cosine and sine, the solutions can be written:

$$
\begin{aligned}
& f_{1}^{\dagger}=\exp (g B)\left[c_{1}^{\dagger} \mathrm{Ch}(\gamma B)+c_{2}^{\dagger} \operatorname{Sh}(\gamma B)\right] \\
& f_{2}^{\dagger}=\exp (g B)\left[c_{1}^{\dagger} \operatorname{Sh}(\gamma B)+c_{2}^{\dagger} \operatorname{Ch}(\gamma B)\right]
\end{aligned}
$$

One also has $f_{i}=\left(f_{i}^{\dagger}\right)^{\dagger}(i=1,2)$. Applying Eqs.6] and 8 to $H_{e l}+H_{e p}+H_{p h}$ yields the "displaced" Hamiltonian, still containing both Fermi and Bose operators. At this point we make the approximation of factorizing the true wave function $|\Psi\rangle$ into the product of Fermi and Bose functions, i.e. $|\Psi\rangle=\left|\Psi_{B}\right\rangle\left|\Phi_{F}\right\rangle$. The Bose operators are now eliminated from the "displaced" Hamiltonian by averaging $e^{\delta R} H e^{-\delta R}$ over a squeezed phonon wavefunction $\left|\Psi_{B}\right\rangle \equiv \exp (-S)\left|0_{p h}\right\rangle$ with $S \equiv$ $\alpha\left(d^{\dagger} d^{\dagger}-d d\right)$ and $d\left|0_{p h}\right\rangle=0$. In the resulting one-particle polaronic Hamiltonian $H^{*} \equiv\left\langle 0_{p h}\left|e^{S} e^{\delta R} H e^{-\delta R} e^{-S}\right| 0_{p h}\right\rangle$ the renormalized interactions read:

$$
\begin{aligned}
& \varepsilon^{*}=\varepsilon-\hbar \Omega \delta(2-\delta)\left(g^{2}+\gamma^{2}\right) \\
& t^{*}=t-2 \hbar \Omega \delta(2-\delta) g \gamma \\
& U^{*}=U\left(3+\tau^{4}\right) / 4+(4 V-J)\left(1-\tau^{4}\right) / 16-2 \hbar \Omega \delta(2-\delta) g^{2} \\
& V^{*}=V\left(7+\tau^{4}\right) / 8+(4 U+3 J)\left(1-\tau^{4}\right) / 32-2 \hbar \Omega \delta(2-\delta)\left(g^{2}-\gamma^{2}\right) \\
& J_{z}^{*}=J\left(5+3 \tau^{4}\right) / 8-(U-V)\left(1-\tau^{4}\right) / 2+4 \hbar \Omega \delta(2-\delta) \gamma^{2} \\
& J_{x y}^{*}=J\left(7+\tau^{4}\right) / 8-(U-V)\left(1-\tau^{4}\right) / 2-2 \hbar \Omega \delta(2-\delta) \gamma^{2} \\
& P^{*}=P\left(9-\tau^{4}\right) / 8-(U-V)\left(1-\tau^{4}\right) / 4-2 \hbar \Omega \delta(2-\delta) \gamma^{2} \\
& X^{*}=\tau X-2 \hbar \Omega \delta(2-\delta) g \gamma
\end{aligned}
$$

where we have defined $\tau \equiv\left\langle 0_{p h}\left|e^{S} \mathrm{Ch}(G B)\right| e^{-S} \mid 0_{p h}\right\rangle=\exp \left[-2 \gamma^{2} \delta^{2} \exp (-4 \alpha)\right]$, and we distinguish the longitudinal $\left(J_{z}^{*}\right)$ and transverse $\left(J_{x y}^{*}\right)$ magnetic couplings. Our results differ qualitatively under several aspects from those following quasi-classical or perturbative treatments $[2]^{-}[4],[6]-[8]$. First, we find that not only the hopping amplitude $t$, but all the interactions, are non-linearly renormalized. Second, if only $\gamma$ is assumed as non-vanishing [4] then $t^{*}=t$ i.e. in the dimer the $S S H$ interaction alone does not modify at all the hopping amplitude. Third, due to $0 . \leq \delta \leq 1$., the cooperative action of both $g$ and $\gamma$ causes $t^{*}<t(<0$.). i.e. it promotes the charge carriers itineracy. 

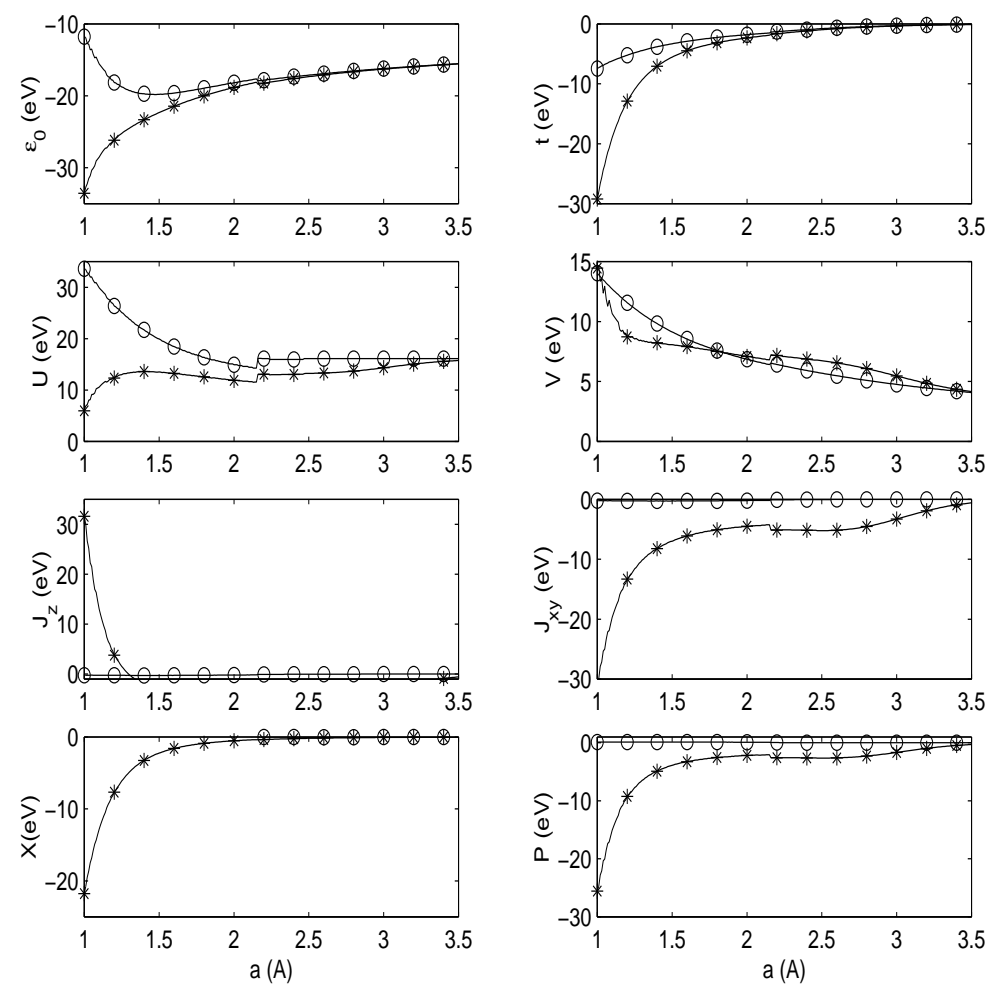

Figure 1: The bare (open dots) and renormalized (asterisks) electronic interactions for the case of two particles. 


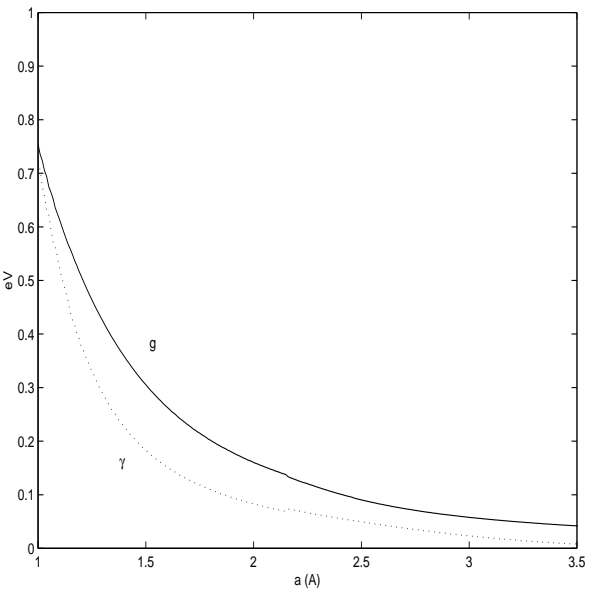

Figure 2: The electron-phonon interactions $g$ (full line) and $\gamma$ (dotted line) vs. $a$.

\section{Numerical results.}

To study the ground state of the system as the dimer length $a$ is varied, we shall adopt the electron interaction parameters $\epsilon, t, X, U, V, J_{z}=J_{x y}=P$ as evaluated as in Ref.11, by assuming a non-degenerate orbital described by Wannier functions built from atomic orbitals of Gaussian shape. Also the variation with $a$ of $g$ and $\gamma$ has been taken into account, following Ref.10. The effective Hamiltonian can be exactly diagonalized 11 yielding the eigenvalues as functions of the variational parameters $\delta, \alpha$ and the Wannier functions shape-defining parameter 11 $\Gamma$. To evaluate the energy of the system, one has to add to $\left\langle\Phi\left|H^{*}\right| \Phi\right\rangle$, the contribution of the squeezed phonons: $E_{p h}=\hbar \Omega\left[\operatorname{Sh}(2 \alpha)^{2}+1 / 2\right]$. From the variational optimization of the total (i.e. electronic plus phononic) energy we obtain the true ground state. For lack of space we discuss only the case of two particles in the orbital.

The bare (open dots) and effective (asterisks) interactions are shown in Fig.1 for a phonon frequency $\hbar \Omega=0.1 \mathrm{eV}$. One notices that only at low $a$ there is a strong renormalisation in the effective interactions. It is due to the fact that, as shown in Fig.2, the electron-phonon interactions decrease with $a$, so that, at low $a$ their renormalizing effect is strong, being weaker for larger $a$. The ground state, in the notation of Ref.11, is the singlet state $|S b\rangle$ for $a<2.16 \AA$, and the $S=0$ component of the triplet $|T, 0\rangle$ for larger $a$. At the transition value of $a$, also $\tau$ and $\Gamma$ vary, indicating that the system interactively readjusts both the phononic and the electronic features.

Knowing the eigenstates $|\Phi\rangle$ of $H^{*}$ we can evaluate the correlation function (CF) $\langle\langle Y\rangle\rangle$ for an operator $Y$ according to $\langle\langle Y\rangle\rangle \equiv\left\langle\Phi\left|\left\langle 0_{p h}\left|e^{S} e^{\delta R} Y e^{-\delta R} e^{-S}\right| 0_{p h}\right\rangle\right| \Phi\right\rangle$. By defining $\theta$ as in Ref.11, one has in the $|S b\rangle$ state:

$$
\begin{aligned}
& \left\langle\left\langle n_{1} n_{2}\right\rangle\right\rangle=\cos ^{2} \theta \\
& \left\langle\left\langle\left(n_{1 \uparrow} n_{1 \downarrow}+n_{2 \uparrow} n_{2 \downarrow}\right)\right\rangle\right\rangle=\left\langle\left\langle\left(c_{1 \uparrow}^{\dagger} c_{1 \downarrow}^{\dagger} c_{2 \downarrow} c_{2 \uparrow}+H . c .\right)\right\rangle\right\rangle=\left[1-\tau^{4} \cos (2 \theta)\right] / 2 \\
& \left\langle\left\langle\left(S_{1}^{+} S_{2}^{-}+S_{1}^{-} S_{2}^{+}\right)\right\rangle\right\rangle=4\left\langle\left\langle S_{1}^{z} S_{2}^{z}\right\rangle\right\rangle=-\left[1+\tau^{4} \cos (2 \theta)\right] / 2
\end{aligned}
$$

Fig. 3 shows the $a$ dependence of the charge distribution CF $\left\langle\left\langle n_{1} n_{2}\right\rangle\right\rangle$, of the transverse magnetic CF $\left\langle\left\langle\left(S_{1}^{+} S_{2}^{-}+\right.\right.\right.$H.c. $\left.\left.)\right\rangle\right\rangle$, from which the longitudinal magnetic CF can be deduced by scaling, and of the on-site bipolaron (OSB) CF $\left\langle\left\langle\left(n_{1 \uparrow} n_{1 \downarrow}+n_{2 \uparrow} n_{2 \downarrow}\right)\right\rangle\right\rangle$ 


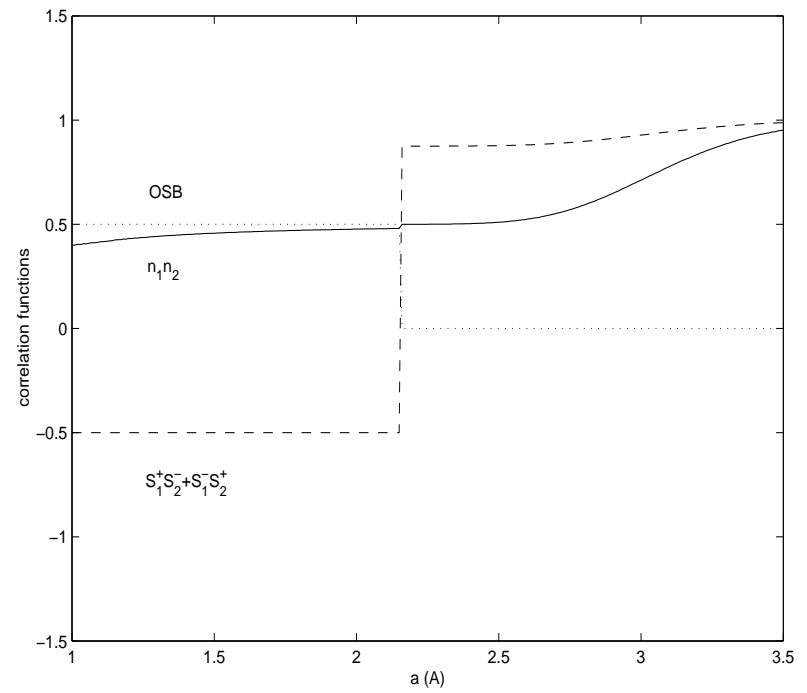

Figure 3: Correlation functions. See the text for explanation.

coinciding with the OSB hopping CF $\left\langle\left\langle\left(c_{1 \uparrow}^{\dagger} c_{1 \downarrow}^{\dagger} c_{2 \downarrow} c_{2 \uparrow}+H . c.\right)\right\rangle\right\rangle$. For $a$ small, where $g$ and $\gamma$ have a strong effect, the formation of an OSB is possible, implying an uneven distribution of the charges between the sites, and, at the same time, the magnetic CF's are sizeable, even though not fully developed. The phonons therefore induce the cohexistence of charge and magnetic correlations for dimer lengths such that the electron-phonon interactions are not negligible, and promote the itineracy of the OSB.

\section{Acknowledgements}

It is a pleasure to thank J.R. Iglesias, M. A. Gusmão, M. Cococcioni, A. Alexandrov, M. Grilli, and particularly A. Painelli, for critical discussions and comments. This work was supported by I.N.F.M. and by MURST 1997 co-funded project "Magnetic Polarons in Manganites".

\section{References}

\section{References}

[1] S. Barisiicet al. Phys. Rev. Lett. 25, 919 (1970).

[2] M. Barma and R. A. Bari, Phys. Rev. B11, 1352 (1975)

[3] L. Pietronero et al. Phys. Rev. B22, 904 (1980).

[4] W. P. Su et al. Phys. Rev B22, 2099 (1980).

[5] E. Fradkin and J. E. Hirsch,Phys. Rev. B27, 1680 (1983). 
[6] B. K. Chackraverty, J. Physique 42, 1351 (1981).

[7] A. L. Kuzemsky et al Physica B 122, 168 (1983); A. L. Kuzemsky and A. P. Zhernov, Int. J. Modern Physics B 4, 1395 (1990).

[8] S. Barisiic et al. Europhys. Letters 3, 1231 (1987); A.A. Aligia et al. Solid State Comm. 65, 501 (1988)]; K. Yonemitsu et al. Phys. Rev.Letters 69965 (1992); A. Smith and P. Hedegård, Phys. Rev. B47, 8039 (1993); S. Ishihara et al.Phys. Rev. B55 , 3163 (1997); M.Capone et al. Phys. Rev. B56, 4484 (1997).

[9] H. Zheng, Phys. Rev. B56 , 14414 (1997).

[10] M. Acquarone and C. Noce, in this Conference.

[11] M.Acquarone et al. Phys. Rev. B58, 7626 (1998). In the Eq.(1) of that paper there is a misprint: the Coulomb interaction $V$ should read $V-J / 2$. 